\title{
Multifrequency Electron Paramagnetic Resonance Characterization of PpoA, a CYP450 Fusion Protein that Catalyzes Fatty Acid Dioxygenation
}

\author{
Alistair J. Fielding, ${ }^{\dagger}$ Florian Brodhun, ${ }^{\ddagger}$ Christian Koch, ${ }^{\ddagger}$ Roberta Pievo, ${ }^{\dagger}$ Vasyl Denysenkov, ${ }^{\perp}$ Ivo Feussner, ${ }^{\ddagger}$ \\ and Marina Bennati ${ }^{*+,}$, \\ ${ }^{+}$Max-Planck Institute for Biophysical Chemistry, Göttingen, Germany \\ ${ }_{\S}^{\S}$ Institute for Organic and Biomolecular Chemistry, Georg-August-University, Göttingen, Germany \\ ${ }^{\ddagger}$ Department of Plant Biochemistry, Albrecht-von-Haller Institute for Plant Sciences, Georg-August-University, Göttingen, Germany \\ ${ }^{\perp}$ Institute for Physical and Theoretical Chemistry, J. W. Goethe University, Frankfurt, Germany
}

Supporting Information

ABSTRACT: PpoA is a fungal dioxygenase that produces hydroxylated fatty acids involved in the regulation of the life cycle and secondary metabolism of Aspergillus nidulans. It was recently proposed that this novel enzyme employs two different heme domains to catalyze two separate reactions: within a heme peroxidase domain, linoleic acid is oxidized to ( $8 R)$-hydroperoxyoctadecadienoic acid $[(8 R)-\mathrm{HPODE}]$; in the second reaction step ( $8 R)$-HPODE is isomerized within a P450 heme thiolate domain to 5,8-dihydroxyoctadecadienoic acid. In the

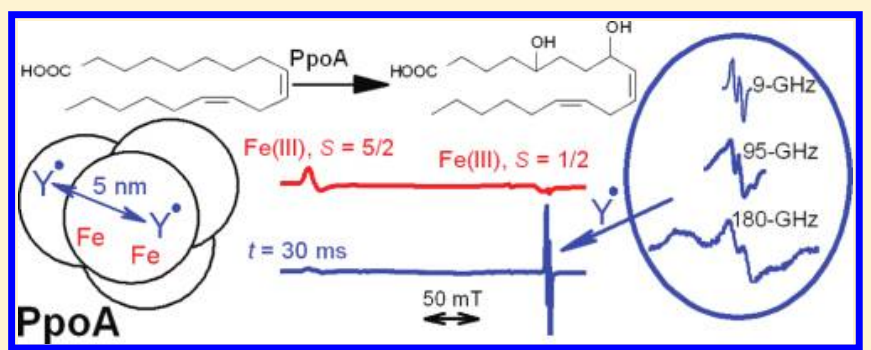
present study, pulsed EPR methods were applied to find spectroscopic evidence for the reaction mechanism, thought to involve paramagnetic intermediates. We observe EPR resonances of two distinct heme centers with $g$-values typical for Fe(III) $S=5 / 2$ highspin (HS) and Fe(III) $S={ }^{1} / 2$ low-spin (LS) hemes. ${ }^{14} \mathrm{~N}$ ENDOR spectroscopy on the $S=3 / 2$ signal reveals resonances consistent with an axial histidine ligation. Reaction of PpoA with the substrate leads to the formation of an amino acid radical on the early millisecond time scale concomitant to a substantial reduction of the $S=5 / 2$ heme signal. High-frequency EPR (95- and 180-GHz) unambiguously identifies the new radical as a tyrosyl, based on $g$-values and hyperfine couplings from spectral simulations. The radical displays enhanced $T_{1}$-spin-lattice relaxation due to the proximity of the heme centers. Further, EPR distance measurements revealed that the radical is distributed among the monomeric subunits of the tetrameric enzyme at a distance of approximately $5 \mathrm{~nm}$. The identification of three active paramagnetic centers involved in the reaction of PpoA supports the previously proposed reaction mechanism based on radical chemistry.

\section{INTRODUCTION}

Heme-containing proteins are responsible for wide ranging biological functions such as oxygen transport, storage, electron transfer and oxygenation reactions. Fatty acid heme dioxygenases convert unsaturated fatty acids to essential molecules for metabolic, protective, developmental, and cell signaling functions. These enzymes are ubiquitously found in animal, fungal and plant kingdoms. ${ }^{1}$ They utilize a catalytic $\mathrm{Fe}$ (III) iron-protoporphyrin and are involved in enzymatic partnerships in which separately expressed fatty acid dioxygenases and cytochrome P450s form bioactive products. ${ }^{2,3}$ A recently discovered hemoprotein, PpoA, has been shown to fuse together fatty acid peroxide production and isomerization in a single protein. ${ }^{4}$

PpoA is one of up to three psi factor (precocious sexual inducer) producing oxygenases (PpoA, $\mathrm{PpoB}$ and $\mathrm{PpoC}$ ) found in Aspergillus nidulans. ${ }^{8-7}$ Psi factors regulate the balance between sexual and asexual life cycles in this fungus as well as secondary metabolism. The genus Aspergillus has been the subject of considerable research because it contains many industrial, medical, and agricultural important species whose mode of reproduction depends primarily on asexual or sexual spore production. ${ }^{8}$ A. nidulans also has been shown to be the cause of respiratory diseases. ${ }^{9,10}$ Deletion of the $p p o A$ and $p p o C$ genes causes a significant effect on the production of secondary metabolites, some of which are carcinogenic. $^{11}$

It has been proposed that the enzyme PpoA harbors two functional heme domains consisting of an $\mathrm{N}$-terminal dioxygenase/peroxidase as well as a C-terminal CYP450 domain. ${ }^{4} \mathrm{UV}$ - vis data of PpoA showed typical Soret bands of ferric hemes; moreover, reduced PpoA reacted with $\mathrm{CO}$ displayed a typical absorption of a heme-CO complex generally known as a characteristic of $\mathrm{P} 450$ heme thiolate enzymes. However, spectroscopic evidence for two distinct hemes as well as their spin states was not available. The biochemical and site-directed

Received: March 10, 2011

Published: May 06, 2011 
Scheme 1. Proposed Catalytic Reaction Scheme of PpoA with LA as a Substrate (as explained in the text)

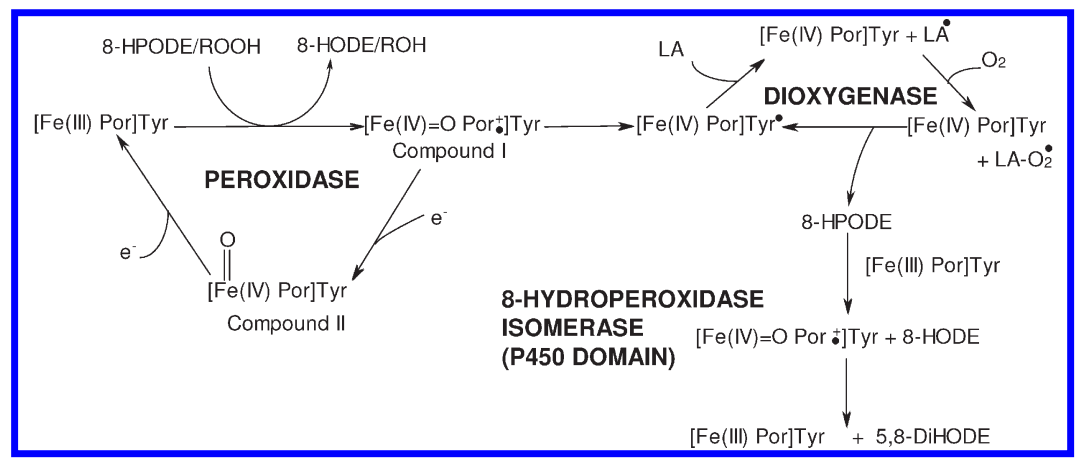

mutagenesis studies suggested that PpoA uses both domains to catalyze two separate reaction steps; the peroxidase domain first oxidizes linoleic acid (LA) to (8R)-hydroperoxy octadecadienoic acid $[(8 R)$-HPODE], then the C-terminal thiolate domain isomerizes ( $8 R)$-HPODE to 5,8-dihydroxyoctadecadienoic acid (5,8-DiHODE).

PpoA shares significant sequence homology with both mammalian prostaglandin $\mathrm{H}$ synthase (PGHS) isoforms, enzymes that are responsible for the first step of biosynthesis of prostaglandins and thromboxanes from arachidonic acid. ${ }^{12,13}$ PGHS is bifunctional and catalyzes the conversion of arachidonic acid into prostaglandin G2 (cyclooxygenase activity) as well as the twoelectron reduction of prostaglandin $\mathrm{G} 2$ into prostaglandin $\mathrm{H} 2$ (peroxidase activity). Identity of conserved catalytic domains ranges from 25 to $29 \%$ for PGHS-2 and $25-26 \%$ for PGHS-1. It had been proposed that reaction of PGHS isoforms 1 or 2 with peroxide generates a radical at $\mathrm{Tyr} 385^{14,15}$ (the numbering refers to the isoenzymes from sheep) that is essential for cyclooxygenase catalysis. ${ }^{16}$ PpoA also shows $42 \%$ amino acid identity with linoleate diol synthase (LDS), which has two enzymatic activities, linoleic acid (8R)-dioxygenase and hydroperoxide isomerase. ${ }^{17}$ LDS has been shown to produce an amino acid radical in a reaction analogous to that of PGHS.

The proposed reaction mechanism of PpoA is based on the widely accepted mechanism of PGHS (Scheme 1). ${ }^{4}$ The initiation step is the generation of the oxoferryl porphyrin cationic radical $\left[\mathrm{Fe}(\mathrm{IV})=\mathrm{O} \mathrm{Por}^{\bullet+}\right]$, often referred to as Compound I, via reduction of in situ hydroperoxide. This species either undergoes intramolecular electron transfer to generate $[\mathrm{Fe}(\mathrm{IV})=\mathrm{O}$ Por $]$ Tyr or is reduced by an exogenous electron donor to $[\mathrm{Fe}(\mathrm{IV})=\mathrm{O}$ Por] (Compound II). The radical species is then used for the abstraction of the hydrogen at C-8 of LA, and subsequent reaction with molecular oxygen generates a peroxy radical which can further abstract a hydrogen atom from a tyrosine to generate ( $8 R)$-HPODE. Isomerization is thought to take place via the intermediate formation of $\left[\mathrm{Fe}(\mathrm{IV})=\mathrm{O} \mathrm{Por}^{\bullet+}\right]$ to give 5,8-DiHODE. First evidence for the proposed radical mechanism and the formation of a carbon-centered radical at C-8 was provided by the analysis of spin-trapping adducts detected by mass spectrometry. ${ }^{4}$ However, this method does not allow detection of protein radicals, which rather requires EPR spectroscopic methods. To date no spectroscopic evidence for any reaction steps is available.

Amino acid radicals are essential in the reactions of many enzymes, ${ }^{18}$ being involved in the formation of cofactors, electron transfer across the protein, or in substrate chemistry. Prototype examples in which the functional role of tyrosyl radicals is well-established includes ribonucleotide reductase (RNR), ${ }^{18,19}$ photosystem $\mathrm{II}^{20}$, and PGHS. ${ }^{14}$ Tyrosyl radicals have also been implicated in other heme-containing peroxidases ${ }^{21}$ and dioxygenases. $^{22,23}$ EPR spectroscopy and particularly high-field $\mathrm{EPR}^{24}$ have emerged as an essential tool to directly detect the formation of protein radicals and to identify the nature of the amino acid site. Furthermore, EPR spectroscopy provides a detailed characterization of the electronic structures of the radical intermediates, which correlates with its redox properties and hydrogen bond interactions. Knowledge of these parameters permits us to rationalize the mechanistic role of the radical.

PpoA is a homotetramer on gel filtration with a subunit molecular mass of about $120 \mathrm{kDa}$. ${ }^{4}$ There is currently no crystal structure available. The aim of this work was to characterize the enzyme in the resting and active state by EPR spectroscopy using samples prepared by rapid freeze-quench and manual mixing conditions. We find evidence for two distinct heme centers and identify a tyrosyl radical generated on reaction of PpoA with either (8R)-HPODE or LA using multifrequency EPR at 9-, 95-, and $180-\mathrm{GHz}$. We observe the co-existence of more than one tyrosyl radical using EPR distance measurements. The work provides spectroscopic evidence for the involvement of paramagnetic centers in the proposed mechanism of PpoA.

\section{MATERIALS AND METHODS}

Materials. LA was purchased from Cayman (Ann Arbor, USA). (8R)-HPODE was produced in $\mathrm{mg}$ amounts by the reaction of PpoA_C1006A or PpoA_H1004A with LA as previously reported. ${ }^{4}$ All other chemicals were purchased from Aldrich at the maximum possible purity.

Sample Preparation. The wild-type and variant forms of PpoA were expressed, isolated, and purified as previously described. ${ }^{4}$ Enzyme concentration was measured by absorption using a calculated $\varepsilon_{280}$ of $126,015 \mathrm{M}^{-1} \mathrm{~cm}^{-1}$ (monomer). In order to avoid significant changes of $\mathrm{pH}$ with certain buffers due to freezing and to aid substrate solubility, HEPES $(50 \mathrm{mM})$ was used for sample preparation. ${ }^{25}$ Spectra at $9-\mathrm{GHz}$ were also obtained in Tris- $\mathrm{HCl}$ buffer $(50 \mathrm{mM})$ and were found to be similar to that recorded using HEPES ( $50 \mathrm{mM})$. For ENDOR measurements, PpoA samples were deuterated by repeated cycles of concentration and resuspension using HEPES $/ \mathrm{D}_{2} \mathrm{O}(50 \mathrm{mM})$, $\mathrm{pD}$ 7.8. Substrates $(1-2 \mu \mathrm{L})$ were added in ethanol (final volume $<2 \%$ ). Protein concentrations of $25-90 \mu \mathrm{M}$ were used for recording the multifrequency EPR spectra.

Rapid freeze-quench (RFQ) samples were prepared by mixing PpoA $(25 \mu \mathrm{M})$ at room temperature with an equal volume of either 160 -fold 
HPODE or LA in a SFM-20 micro-FQ instrument interfaced with MPS32 software (BioLogic) and shot into a liquid-nitrogen-cooled isopentane bath $\left(-140^{\circ} \mathrm{C}\right)$. Aging times were determined by the ram speed, aging tubing volume, $1 \mathrm{~cm}$ flight between nozzle and bath, and the freezing time. Frozen samples were then packed with a Teflon-tipped steel rod into $4 \mathrm{~mm}$ quartz EPR tubes. Control samples were mixed with equivolumes of buffer. All samples were prepared under aerobic conditions and were flushed with helium after freezing to remove molecular oxygen. Calibration of the apparatus was achieved using the known rate constant for the reaction of equine skeletal muscle met-myoglobin with sodium azide in $20 \mathrm{mM}$ TRIS, $\mathrm{pH} 7.8,0.1 \mathrm{~N}$ in $\mathrm{NaNO}_{3} .{ }^{26} \mathrm{~A}$ packing factor of 0.5 was used to calculate concentrations. For manual mixing, samples were prepared by mixing $50 \mu \mathrm{L}$ of the native enzyme with an equivolume of 160-fold excess of either (8R)-HPODE or LA (buffered at the same $\mathrm{pH}$ as the sample), directly in the 4-mm EPR tubes. The reaction was stopped by rapid immersion of the EPR tube into a dry ice/ethanol bath and then transferred to liquid nitrogen.

9-GHz EPR Spectroscopy. Continuous-wave (CW) EPR spectra were recorded using a Bruker EMX spectrometer and a Bruker ER041 bridge. The spectrometer was equipped with a super high sensitivity probe head and a liquid helium cryostat (Oxford Instruments). Spin concentrations of the high-spin (HS) and low-spin (LS) ferric heme were estimated by double integration of the CW-EPR signal ${ }^{27}$ and comparison with either HS ferric met-myoglobin $(1.0 \mathrm{mM})$ or metmyoglobin $(1.0 \mathrm{mM})$ mixed with 40 -fold azide, which generates $100 \%$ LS heme. The concentration of radical signals was estimated against several standards, such as a known concentration of stable tyrosyl radical of subunit $\beta$-2 in class I RNR from Escherichia coli, nitroxide radical standards, and CuEDTA. The overall error in the quantitation is estimated to be up to $20 \%$. EPR conditions are stated on the figure legend. EPR simulations were performed using the EasySpin (3.1.0) EPR simulation package. ${ }^{28}$ The rms of the residuals was kept below 0.12 .

Pulse EPR experiments were performed on a Bruker ELEXSYS E580 with a Bruker dielectric resonator and an Oxford CF 935 cryostat. Pulse lengths and pulse spacing were adjusted for each experiment, depending of the type of paramagnetic species observed. Davies ENDOR was performed using the pulse sequence $\pi-T-\pi / 2-\tau-\pi-\tau$-echo, with microwave pulse lengths of 48,24 , and 48 ns, respectively. Microwave power was adjusted to optimize the echo intensity at the given pulse lengths for HS vs LS heme signals. Field positions for ENDOR excitation are noted in the figure captions. The field position used for PpoA at $B \| g_{z}$ contains contributions of both LS and HS ferric signals and the pulses as well as shot repetition times were set on the basis of the values optimized for purely HS met-myoglobin under identical experimental conditions (temperature, field position, resonator coupling). RF pulse power of about $500 \mathrm{~W}$ was produced by a $1.2 \mathrm{~kW}$ RF amplifier from Barthel. Further experimental details are given in the figure captions.

Distance Measurements. Double electron electron resonance (DEER), often referred to as PELDOR (pulsed electron-electron double resonance $)^{29-31}$ measurements were performed using the four-pulse sequence $\pi / 2_{v_{\text {obs }}}-\tau_{1}-\pi_{v_{\text {obs }}}-t^{\prime}-\pi_{v_{\text {pump }}}-\left(\tau_{1}+\tau_{2}-\mathrm{t}^{\prime}\right)-\pi_{v_{\text {obs }}}-\tau_{2}$-echo. ${ }^{32}$ Background echo decay was corrected using a mono-exponential function. The frequency of the signal modulation for the dipolar interaction between the spin probes $g_{\mathrm{A}}$ can be related to the interspin distance by using the following eq 1 :

$$
v_{\mathrm{dd}}=\frac{\mu_{0} \mu_{\beta}^{2} g_{\mathrm{A}} g_{\mathrm{B}}}{4 \pi h} \frac{\left(3 \cos ^{2} \theta-1\right)}{r_{\mathrm{AB}}^{3}}
$$

where $v_{\mathrm{dd}}$ is the modulation frequency, $\mu_{0}$ is the vacuum permeability, $\mu_{\beta}$ is the Bohr magneton, $h$ is the Plank constant, $g_{\mathrm{A}}$ and $g_{\mathrm{B}}$ are the $g$-values, and $r_{\mathrm{AB}}$ is the interspin distance. We use an average $g$-value of 2.004 for both $g_{\mathrm{A}}$ and $g_{\mathrm{B}}$. The distance distribution was calculated by fitting the corrected dipolar evolution data using Tikhonov regularization as implemented in the

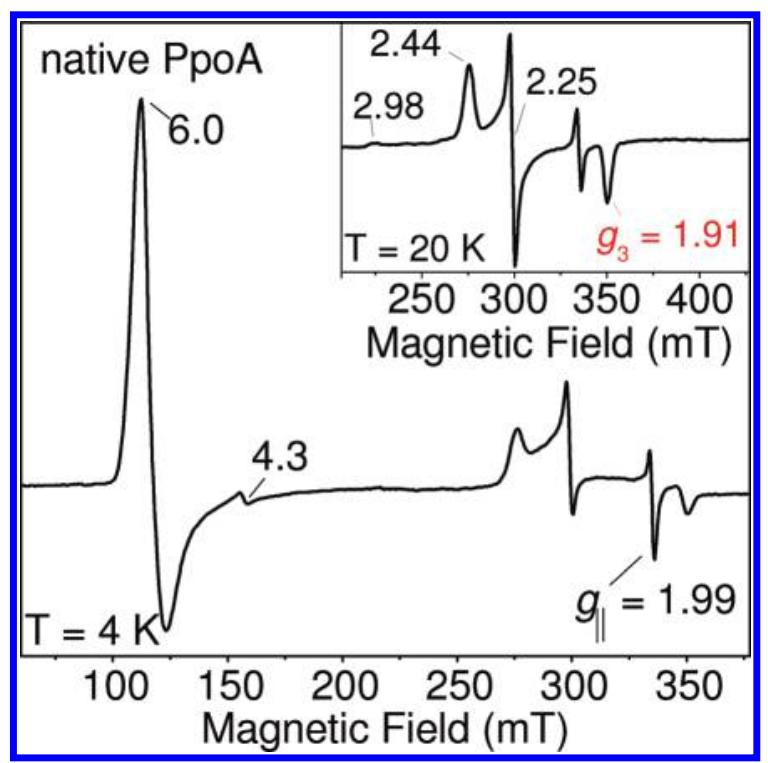

Figure 1. 9-GHz CW EPR spectra of PpoA $(75 \mu \mathrm{M})$ in HEPES buffer $\mathrm{pH} 7.4$ recorded at $4.3 \mathrm{~K}, 4 \mathrm{G}$ MA (modulation amplitude), $P$ (microwave power $)=1 \mathrm{~mW}, \mathrm{MF}$ (modulation frequency $)=100 \mathrm{kHz}, 1$ scan. Inset: 9-GHz EPR spectra of the LS species of PpoA recorded at $20 \mathrm{~K}$, $P=0.05 \mathrm{~mW}, \mathrm{MA}=7 \mathrm{G}$ and 4 scans; $g$-values are labeled.

DeerAnalysis2008.1 package. ${ }^{33}$ Further details about the experiments are provided in the figures. The phase memory time, $T_{\mathrm{m}}$ of the radical species was found to be $\sim 1 \mu \mathrm{s}$ at $5 \mathrm{~K}$ and 9-GHz. It was not possible to significantly raise $T_{\mathrm{m}}$ in deuterated solvent or glycerol and lengthen the acquisition window.

High-Frequency (HF) EPR Spectroscopy. Continuous-wave 94-GHz and pulse EPR experiments were performed by using a Bruker Elexsys 680 spectrometer (Power Upgrade 2). EPR conditions are stated on the figure legends. The microwave frequency was measured by a built-in frequency counter, and the magnetic field was calibrated by using a separate sample of 1,3-bis-diphenylene-2-phenylallyl (BDPA, $g=2.0026)^{34}$ doped into polystyrene. Samples were mixed into $0.9 \mathrm{~mm}$ o.d. (outer diameter), $0.5 \mathrm{~mm}$ i.d., (inner diameter) quartz capillaries.

Field-swept (180-GHz) echo-spin echo (ESE)-detected spectra were recorded on a home-built spectrometer previously reported with typical $\pi / 2$ pulse lengths of $40 \mathrm{~ns}^{35}$ Samples were mixed into $0.55 \mathrm{~mm}$ o.d. and $0.4 \mathrm{~mm}$ i.d. quartz capillaries. The field was calibrated against a separate sample of manganese/magnesium oxide ( $\mathrm{Mn}$ (II)/ $\mathrm{MgO})$ standard $(g=2.00101){ }^{36}$ The absolute error in $g$-values was about $1 \times 10^{-4}$.

\section{RESULTS}

EPR Spectra of the Ferric Heme Groups of PpoA. We have used 9-GHz CW EPR spectroscopy to investigate the oxidation and spin state of the heme groups in PpoA. Figure 1A displays the full-scan native spectrum of resting PpoA at $4.3 \mathrm{~K}$. The spectrum exhibits turning points at $g_{\perp}{ }^{\text {eff }} \approx 6.0$ and $g_{\|}{ }^{\text {eff }} \approx 2$ typical for HS ferric $\left[\mathrm{Fe}(\mathrm{III}), S={ }^{5} / 2\right]$ heme. ${ }^{37}$ The HS resonances contributed a factor of about 3.5 over the total protein concentration, equivalent to $0.9 \mathrm{Fe} /$ monomer. The feature observed at $g=$ 4.3 was assigned to iron impurities and not considered in this study. Another intensive resonance was observed with turning points at $g_{1}=2.43, g_{2}=2.25$, and $g_{3}=1.91$ (see Figure 1 inset, spectrum at $20 \mathrm{~K}$ under nonsaturating conditions) typical for LS ferric heme $[\mathrm{Fe}(\mathrm{III}), S=1 / 2] .{ }^{38}$ This LS resonance contributed a factor of about 3 over the total protein concentration, 
equivalent to $0.8 \mathrm{Fe} /$ monomer. The LS $g$-values are consistent with those found in $\mathrm{P} 450$ proteins such as $\mathrm{P} 450_{\mathrm{CAM}}$ that have cysteine and water as axial ligands for the heme core (see also Table S1). ${ }^{38,39 a}$ There was no detection of a corresponding HS state of P450 enzymes with $g$-values at $7.9(\sim 84 \mathrm{mT}), 4.9$, and 1.8 , usually associated to substrate binding. ${ }^{40}$ The spectrum at $20 \mathrm{~K}$ under nonsaturating conditions also displays weak features of a second LS signal with $g_{1} \approx 2.98$, and $g_{3} \geq 1$.7. The intensity of this species represents about $15 \%$ of the dominant LS signal. An asymmetric signal at $g=2$ is observed that we assign to the overlap of the $g_{\|}{ }^{\text {eff }} \approx 2 \mathrm{HS}$ signal and a weak radical signal $(<5 \%)$.

ENDOR Reveals Histidyl Ligation to the HS Heme. In order to verify the hypothesis proposed in ref. ${ }^{4}$ that the enzyme contains two distinct heme domains, particularly the presence of a peroxidase type of domain with a penta-coordinated heme and a histidyl axial ligand, we have employed ${ }^{14} \mathrm{~N}$ ENDOR to discern hyperfine couplings ( $h f \mathrm{c}$ ) of a histidine ligand. Seminal ${ }^{14} \mathrm{~N}$-ENDOR work by Feher and co-workers ${ }^{41}$ had demonstrated that axial histidine hfcs in HS heme proteins are well-resolved in ENDOR at field excitation positions close to $B \| g_{z}$, where the four strongly coupled ${ }^{14} \mathrm{~N}$ nuclei of the heme porphyrin ring become almost magnetically equivalent. Furthermore, at $B \| g_{z}$ orientation selective ENDOR allows recording single-crystal-like spectra. ENDOR has since been used to confirm the presence or absence of an axial nitrogen ligands in other heme complexes. ${ }^{42,43}$

${ }^{14} \mathrm{~N}$-Davies ENDOR spectra $(9-\mathrm{GHz})$ of PpoA were recorded at field positions corresponding to $B \| g_{z}$ of the HS and $B \| g_{3}$ of LS heme signals, respectively, (Figure 2 and Figure S1) and compared with ENDOR spectra of model high-spin heme proteins with and without a histidyl ligand, such as met-myoglobin (B) and hemin $(C) \cdot{ }^{40,41}$ All spectra are characterized by a doublet of doublets in the region between approximately 2 and $6 \mathrm{MHz}$. The main doublet (centered between 3.5 and $4 \mathrm{MHz}$ ) is separated by about twice the Larmor frequency of ${ }^{14} \mathrm{~N}\left(v_{\mathrm{N}}\right)$, i.e. $\approx 2 \mathrm{MHz}$ at $\mathrm{X}$-band. The shape of the spectra is typical for quadrupole nuclei with hfc $\gg v_{\mathrm{N}}$, for which the center of the 'doublet of doublets' reflects the orientation dependent $A / 2$ value. The intensity and the sharpness of the spectra in this region are very consistent with spectra previously reported for magnetically equivalent porphyrin nitrogens. ${ }^{41}$ The comparison with the reference spectra $(B, C)$ and the simulations support this assignment.

Nevertheless, spectra (A) of PpoA and (B) of met-myoglobin clearly show an additional peak at $8-8.5 \mathrm{MHz}$ and a peak/ shoulder at $\sim 6.3 \mathrm{MHz}$. In the latter, these resonances have been previously assigned to the high-frequency doublet (see splitting pattern in Figure 2) of an axial histidine ligand. ${ }^{41}$ The compelling similarity of (B) and the PpoA spectrum (A) strongly suggests a similar assignment for PpoA. These resonances are clearly absent in the spectrum of hemin (C), used as a reference for a HS heme without histidyl ligation and furthermore in the spectrum of PpoA (D) when shifting the field from $B \| g_{z}$ of HS heme to $B \| g_{3}$ of LS heme. Spectrum (D) was recorded by optimizing the experimental parameters for a LS heme; however, a spectrum at the same field position with parameters optimized for HS heme showed almost no resonances (Figure S2, D). Also, spectra recorded at $B \| g_{z}$ of HS using parameters optimized for LS heme show only weak resonances because of low spectral intensity of LS at this field position (Figure S2, C). Therefore, our results provide evidence that the 6 and $8 \mathrm{MHz}$ features in (A) arise solely from the HS center. The possibility that the peak at $8 \mathrm{MHz}$ in (A) could arise from an exchangeable proton instead of a fifth nitrogen was excluded by a control experiment in deuterated buffer (Figure S2, B).

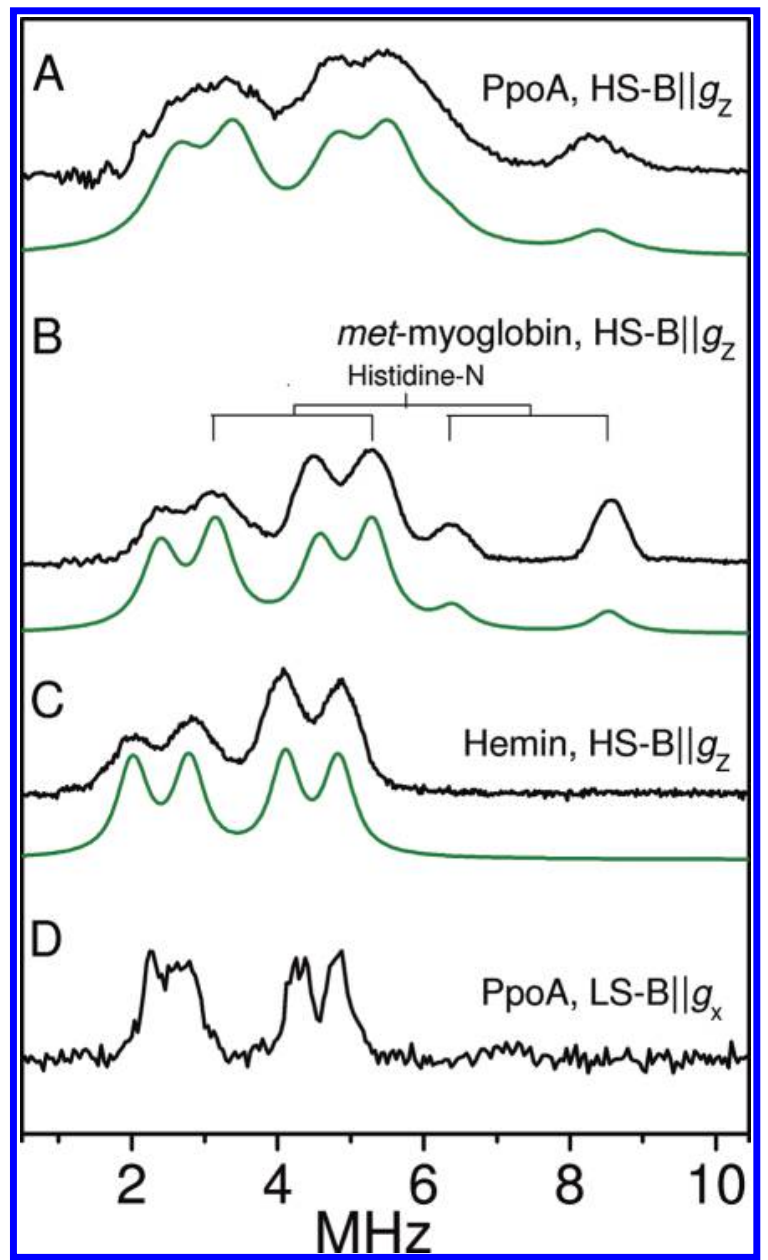

Figure 2. 9-GHz Davies-ENDOR spectra taken at $3.8 \mathrm{~K}$. The experimental parameters: $\pi / 2=24 \mathrm{~ns} ; \pi=48 \mathrm{~ns} ; \tau=240 \mathrm{~ns}$. Shot repetition time $=2.5 \mathrm{~ms}$; shots $/$ point $=60$; RF pulse $=2 \mu \mathrm{s}$. (A) PpoA $0.3 \mathrm{mM}$ in $50 \mathrm{mM}$ HEPES solution $\mathrm{pH} 7.4$ at $347.2 \mathrm{~m}, 888$ scans. (B) met-myoglobin $6.0 \mathrm{mM}$ at $347.2 \mathrm{mT}$ in $50 \mathrm{mM}$ HEPES solution $\mathrm{pH}$ 7.4: $50 \%$ glycerol solution, 22 scans. (C) Hemin $5.0 \mathrm{mM}$ at $347.2 \mathrm{mT}$ in diethylformamide:ethanol, 28 scans. (D) PpoA $0.3 \mathrm{mM}$ in $50 \mathrm{mM}$ HEPES solution $\mathrm{pH} 7.4$ at $361.1 \mathrm{mT}$ (LS, $g_{3}=1.91$ ) using a shot repetition time $=50 \mathrm{~ms}$, RF pulse $=15 \mu \mathrm{s}$, shots $/$ point $=10,325$ scans. Simulations are shown in green (Table S1).

The ENDOR spectra were simulated using our own software ${ }^{34}$ and analytical solutions for the ${ }^{14} \mathrm{~N}$ ENDOR frequencies at $B \| g_{z}$ in HS hemes from second-order perturbation theory. ${ }^{41} \mathrm{We}$ first performed simulations of met-myoglobin and hemin spectra using hfc and quadrupole values from the literature ${ }^{41,42,44}$ (Table S1) and assuming collinear $g$, hyperfine and quadrupole tensors. ${ }^{41}$ As observed in ref., ${ }^{41}$ we also found that deviation from first-order resonance frequencies is less than $\sim 0.2 \mathrm{MHz}$. Spectrum (A) was simulated with five ${ }^{14} \mathrm{~N}$ nuclei and a slight inequivalency of the porphyrin hfcs $\left(A_{z}=7.9\right.$ and $8.1 \mathrm{MHz}$ respectively), which causes the observed broadening. The fifth hfc resulted in $11.9 \mathrm{MHz}$ as compared to $11.5 \mathrm{MHz}$ in metmyoglobin and was assigned to a histidine axial ligand. The hyperfine values and quadrupole couplings from our simulations and from the literature are summarized in Table S1.

Generation of a Radical Signal. The reaction of PpoA with (8R)-HPODE and LA was studied with 9-GHz EPR. Rapid freeze-quench samples were collected after aging of PpoA 


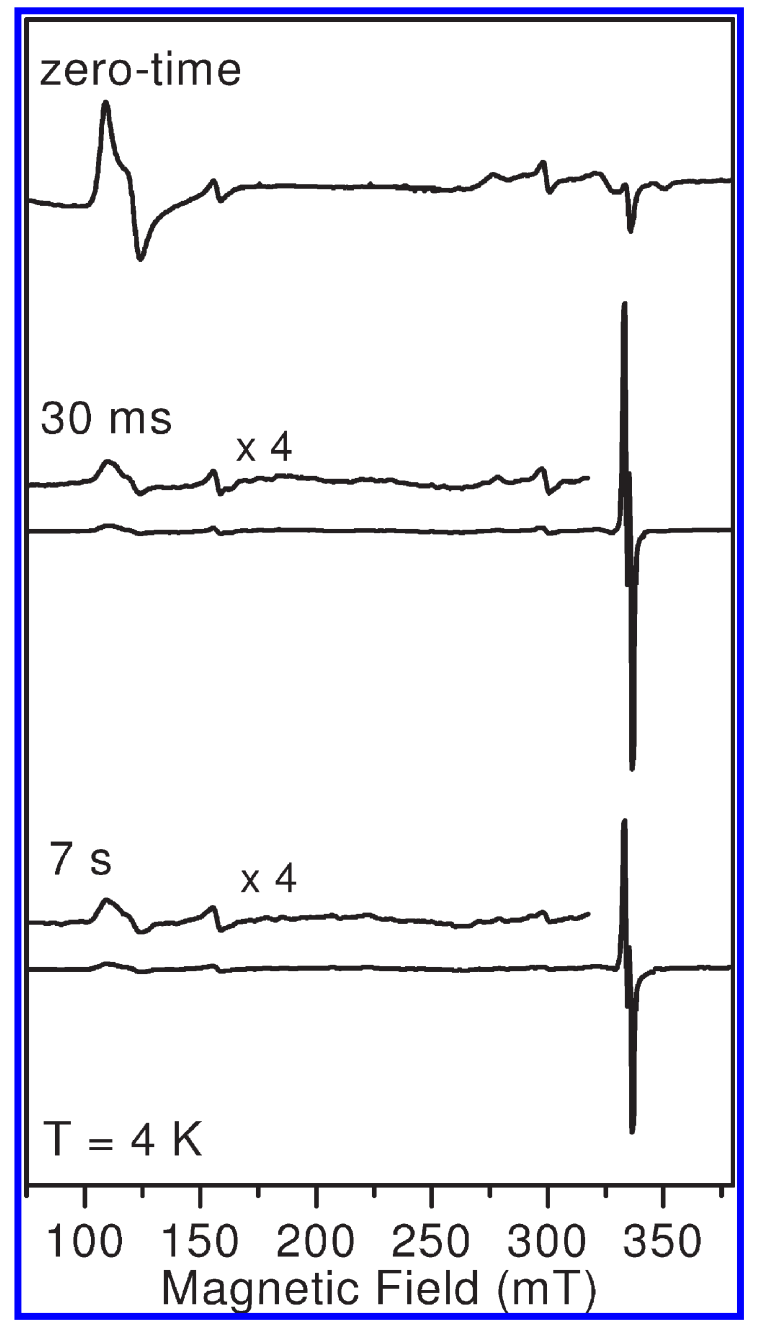

Figure 3. 9-GHz CW EPR spectra of the reaction between PpoA $(25 \mu \mathrm{M})$ and 160-fold (8R)-HPODE in HEPES buffer $\mathrm{pH} 7.4$ using aging times of $30 \mathrm{~ms}$ and $7 \mathrm{~s}$. PpoA mixed with buffer using an aging time of $30 \mathrm{~ms}$ is shown as a reference (zero time). Spectra were recorded at $4 \mathrm{~K}, \mathrm{MA}=4 \mathrm{G}, P=1.0 \mathrm{~mW}, 100 \mathrm{kHz}, 16$ scans.

$(25 \mu \mathrm{M})$ with a 160 -fold excess of (8R)-HPODE or alternatively LA between $30 \mathrm{~ms}$ and $7 \mathrm{~s}$. Manually mixed samples were also prepared for comparison using an aging time of $7 \mathrm{~s}$. The $9-\mathrm{GHz}$ spectra of the reaction mixture revealed changes of the iron oxidation state and the generation of a radical species. Representative spectra of the reaction with ( $8 R$ )-HPODE taken at $30 \mathrm{~ms}$ and $7 \mathrm{~s}$ are shown in Figure 3. The spectrum at $30 \mathrm{~ms}$ aging time showed a strong reduction in the intensity of the HS ferric heme to $<20 \%$ percent of the initial amount. Concomitantly, a new strong radical signal at $g_{a v}=2.004$ appeared. The radical signal was estimated to represent $\sim 1.6$ spins/tetramer. There was no change within experimental error of the intensity of the LS signal at $20 \mathrm{~K}$ under nonsaturating conditions. At the later time of $7 \mathrm{~s}$, a reduction (40\%) of the radical and the LS ferric heme signal was observed. A full set of data recorded at different time points between $30 \mathrm{~ms}$ and $7 \mathrm{~s}$ and also under nonsaturating conditions for the LS heme is displayed in SI (Figure S3). The radical in all samples had a peak-to-trough of $34 \mathrm{G}$ and a distinctive "doublet shape" (Figure 4). There were also no significant changes in line shape comparing the radical formed on the millisecond scale to that formed on the later time points

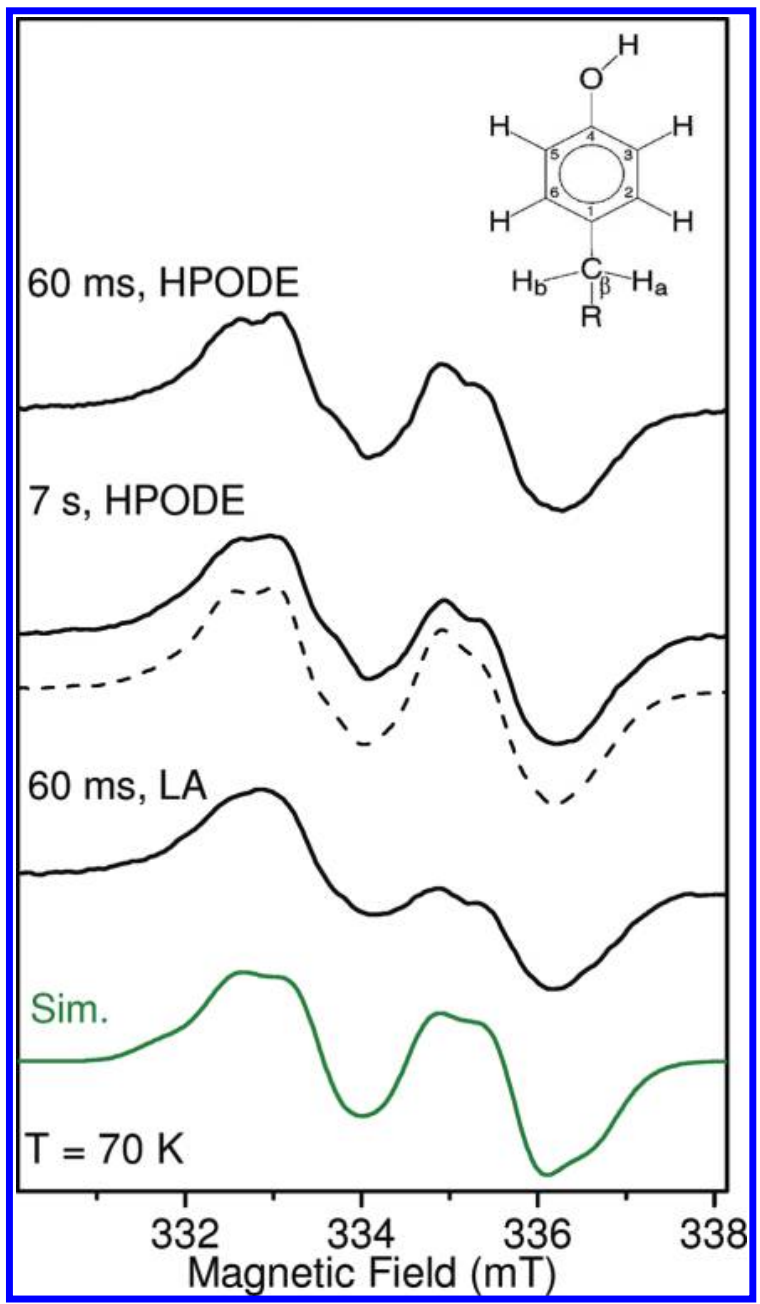

Figure 4. 9-GHz CW spectrum at $70 \mathrm{~K}$ of the radical formed after reaction of PpoA (25 $\mu \mathrm{M})$ with 160 -fold excess (8R)-HPODE using aging times of $60 \mathrm{~ms}$ and $7 \mathrm{~s}$. Spectrum of a $7 \mathrm{~s}$ manually mixed sample is shown in black dashes. Comparison with reaction of PpoA with 160 -fold LA using an aging time of $60 \mathrm{~ms}$. Spectra were recorded with a $M A=2 \mathrm{G}$, $P=0.0127 \mathrm{~mW}$ and $\mathrm{MF}=100 \mathrm{kHz}, 4-100$ scans. Spectra are normalized to one. Simulation is shown in green. Inset: Tyrosine numbering scheme.

(Figure 4). When the experiments were repeated using LA (Figure S4), a radical signal was observed of similar line shape (Figure 4). The magnitude of the radical signal was $\sim 80-90 \%$ less than that using ( $8 R$ )-HPODE, concomitant to $\sim 50 \%$ reduction in the $H S$ intensity. We note that the slight asymmetric line shape of the radical spectrum produced by the reaction of PpoA with LA is caused by the weakness of the radical signal as compared with the background signals of the hemes. The results are consistent with the proposed mechanism in Scheme 1, according to which, in this latter case, the reaction is lacking the fatty acid hydroperoxide as activator and is thought to be initiated by low amounts of hydroperoxide impurities, producing in turn only a low amount of radical species.

EPR Chacterization of Mutants Y374F. Previous biochemical studies by Brodhun et al. ${ }^{4}$ had shown that the mutant Y374F is lacking activity with LA; however, when the mutant was incubated with the intermediate product $(8 R)-\mathrm{HPODE}$, it was able to 


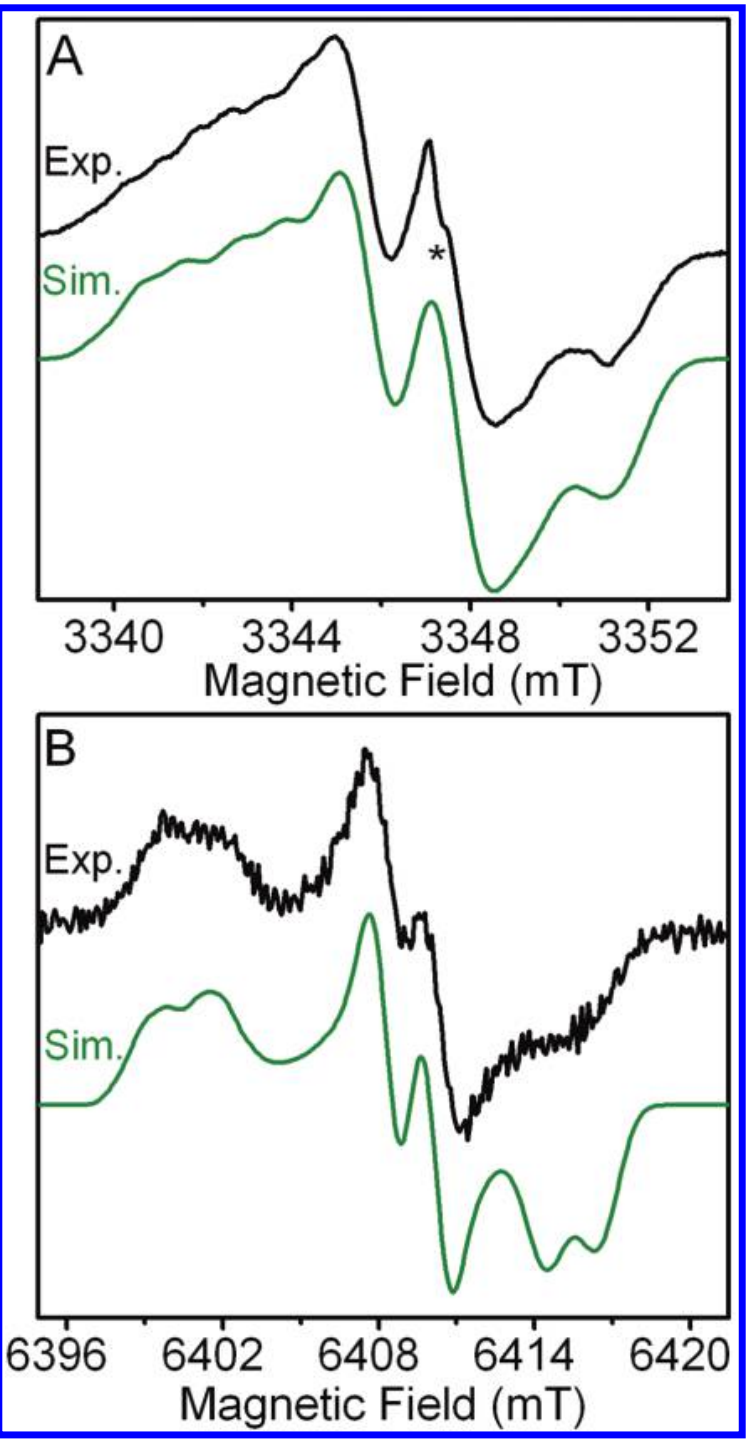

Figure 5. (A) 94-GHz CW spectrum of the radical generated after reaction of PpoA with 160-fold excess (8R)-HPODE for $7 \mathrm{~s}$ (black). Spectrum was recorded at $90 \mathrm{~K}, \mathrm{MA}=2 \mathrm{G}, P=0.005 \mathrm{~mW}, 100 \mathrm{kHz}$, 4 scans. (B) $180-\mathrm{GHz}$ Echo-detected spectrum (1st derivative) of the radical after reaction with 160 -fold excess (8R)-HPODE for $7 \mathrm{~s}$ (black). The experimental parameters: $\pi / 2=40 \mathrm{~ns}, \tau=200 \mathrm{~ns}, \pi=80 \mathrm{~ns}$; shot repetition time $=20 \mathrm{~ms}$; shots $/$ point $=40$; scans $=700 ; T=10 \mathrm{~K}$. Simulations using the parameters given in Table 1 are shown in green. * Clipping is caused by manganese impurities.

catalyze the formation of 5,8-DiHODE. The results suggested that Y374 is not involved in the isomerase reaction of Scheme 1 but rather in substrate dioxygenation. Mutation of Y374 in the proposed peroxidase domain to $\mathrm{Y} 374 \mathrm{~F}$ was based on sequence alignment with Y385 in sheep PGHS-1, a residue which is necessary for cyclooxygenase activity. ${ }^{16}$ The EPR spectra of substrate-free mutant Y374F at 4 and $20 \mathrm{~K}$ showed the presence of both HS and LS ferric heme with $g$-values similar to those of the native enzyme (Figure S5). Repetition of a manual mixing experiment at $3 \mathrm{~s}$ with $\mathrm{Y} 374 \mathrm{~F}$ and 160 -fold (8R)-HPODE showed no generation of a radical (Figure S5). These findings were consistent with the previously observed absence of reactivity with LA in biochemical studies ${ }^{4}$ and demonstrate the proposed role of the radical as mediator, essential for substrate oxidation.
Table 1. Parameters Used To Simulate the 9-, 94-, and $180-\mathrm{GHz}$ EPR Spectra of the Tyrosyl Radical in the Reaction of PpoA with 160-fold (8R)-HPODE

\begin{tabular}{|c|c|}
\hline omponent $^{a}$ & value \\
\hline \multicolumn{2}{|l|}{$g$-values } \\
\hline$g_{x}\left(\sigma_{x}\right)^{b}$ & $2.0069(0.0005)$ \\
\hline$g_{y}$ & 2.0042 \\
\hline$g_{z}$ & 2.0022 \\
\hline \multicolumn{2}{|l|}{$\mathrm{H}_{\mathrm{ssl}}{ }^{c}$} \\
\hline$A_{x}$ & 63.8 \\
\hline$A_{y}$ & 52.4 \\
\hline$A_{z}$ & 54.4 \\
\hline \multicolumn{2}{|l|}{$\mathrm{H}_{\mathrm{A}}$} \\
\hline$A_{x}$ & 20.7 \\
\hline$A_{y}$ & 13.6 \\
\hline$A_{z}$ & 16.4 \\
\hline \multicolumn{2}{|l|}{$\mathrm{H}_{\mathrm{B}}$} \\
\hline$A_{x}$ & 25.8 \\
\hline$A_{y}$ & 12.5 \\
\hline$A_{z}$ & 18.7 \\
\hline \multicolumn{2}{|l|}{$\mathrm{H}_{\mathrm{C}}$} \\
\hline$A_{x}$ & 26.9 \\
\hline$A_{y}$ & 7.1 \\
\hline$A_{z}$ & 16.1 \\
\hline
\end{tabular}

${ }^{a}$ Absolute values for hyperfine coupling constants are given in megahertz. Line widths of 5.6, 6, and $7 \mathrm{G}$ were used at 9-, 94-, and 180-GHz, respectively. ${ }^{b}\left(\sigma_{i}\right)$ is the half-width at half-maximum of the Gaussian distribution about $g_{i}$ (Easyspin $g$ Strain function with a broadening tensor collinear to the $\mathrm{g}$-tensor). ${ }^{c}$ In the simulations, the $g$ and hyperfine tensors of the $\beta$-methylene protons were assumed as collinear. For the 3,5-ring protons, an Euler angle $\alpha= \pm 25^{\circ}$ was used for $\mathrm{H}_{\mathrm{B}}$ and $\mathrm{H}_{\mathrm{C}}$.

Identification of a Tyrosyl Radical by Multifrequency EPR. High-field EPR was performed in order to identify the radical species at $g=2$ generated during reaction of PpoA with substrate. Figure 5 compares EPR spectra at 94- and 180-GHz after $7 \mathrm{~s}$ of mixing with ( $8 R$ )-HPODE. Experiments with LA gave very similar spectra (Figure S6). From the $94-\mathrm{GHz}$ spectrum it was possible to discern additional features as compared to the $9-\mathrm{GHz}$ spectrum due to the contribution of the anisotropic g-tensor. However, the line shape at $94-\mathrm{GHz}$ still results as a complex combination of hyperfine and g-tensor anisotropy. In order to fully resolve the g-tensor we performed the experiment at 180-GHz. The derivative of the echo-detected EPR spectrum at $10 \mathrm{~K}$ (Figure 5B) showed a typical line shape dominated by $g$-anisotropy. A discernible hyperfine splitting is observed at $g_{x}$ and $g_{y}$, but the $g_{z}$ edge is more broadened. In our simulation strategy we have attempted to determine as many parameters as possible independently; therefore, we started with a simulation of the $180-\mathrm{GHz}$ spectrum, which depends predominantly only on the $g$-values and the large visible hyperfine splitting. The simulation displayed in Figure 5B led to a g-tensor of $g_{x}=2.00695, g_{y}=2.0042$ and $g_{z}=2.0022$ and a large hyperfine coupling, the later being refined by the simulation of the $9-\mathrm{GHz}$ spectrum as discussed below. The $g$-anisotropy for the radical $\left(g_{x}-g_{z}=0.0048\right)$ is within the range of typical tyrosyl radicals $\left(g_{x}-g_{z} \sim 0.007-0.004\right) .{ }^{45-47}$ Furthermore, the line shape of the observed high-field EPR 
spectra provides a fingerprint for a tyrosyl radical. Indeed, it displays a large isotropic splitting at all three $g$-values superimposed by an anisotropic hyperfine substructure. The latter was visible in the $94-\mathrm{GHz}$ spectrum at the low-field side and notably arises from the 3,5-ring protons. ${ }^{34}$

The 9- and 94-GHz spectra were simulated using the aforementioned $g$-values and hyperfine couplings consistent with that of tyrosyl radicals. The best parameters found to reproduce the complementary multifrequency spectra at 9-, 94-, and $180-\mathrm{GHz}$ are given in Table 1 . The simulation required four nonequivalent hyperfine tensors, one of which was substantially larger, with the highest hyperfine component $A_{x}=63.8 \mathrm{MHz}$ and an anisotropy of $\Delta A \approx 11 \mathrm{MHz}$. We assigned this tensor to the coupling of one of the $\beta$-methylene protons (Table 1 ). The three other tensors had similar sizes, with the largest values varying between 20 and $27 \mathrm{MHz}$. These tensors were assigned to the 3,5-ring and the second $\beta$-methylene proton, as in tyrosyl radicals the coupling to the other 2,6-ring protons is much smaller. ${ }^{34}$ The value of the second $\beta$-methylene proton is similar to that previously observed for mouse RNR ${ }^{47}$ A more precise assignment of either one of these tensors to one of the mentioned protons is difficult. Nevertheless, the results suggest that the couplings of the 3, 5-ring protons were slightly nonequivalent, which has also been observed previously in mouse $\mathrm{RNR}^{47}$ and associated with the presence of a hydrogen bond (see Discussion section).

Interestingly, the $180-\mathrm{GHz}$ ESE spectrum at $10 \mathrm{~K}$ showed weak broadening along the $g_{x}$ that was reproduced in the simulation through a Gaussian broadening of the $g_{x}$ value. This broadening is indicative for a heterogeneity in the electrostatic environment (see also Discussion) or alternatively for the coexistence of more than one tyrosyl radical. We observed also some broadening in the $g_{z}$ axis of the spectrum as compared to the simulation. The effect of anisotropic $T_{1,2}$ relaxation or distribution in $g$-values/hyperfine splitting may account for the latter broadening and are expected to be more pronounced in the $180-\mathrm{GHz}$ rather than in the $94-\mathrm{GHz}$ EPR frequencies.

The hyperfine tensors of Table 1 allow an estimate of the dihedral angle $\theta$ to the protein backbone, defined by the direction of the $p_{z}$ axis on $\mathrm{C}_{1}$, the $\mathrm{C}_{1}-\mathrm{C}_{\beta}$ bond, and the direction of either one of the $\beta$-methlyene protons. Using the McConnell relation (eq 2): ${ }^{48-50}$

$$
A_{\text {iso }}=\rho_{\mathrm{C} 1}\left(B_{0}+B_{1} \cos ^{2} \theta\right)
$$

where $A_{\text {iso }}$ is the isotropic proton hyperfine coupling constant arising from the $\mathrm{C}_{\mathrm{ss}}$ hydrogen, $\rho_{\mathrm{C} 1}$ is the spin density at $\mathrm{C}_{1}$ of the phenoxyl ring, $\theta$ is the dihedral angle, and $B_{0}$ and $B_{1}$ are empirical constants $\left(B_{0}=1-2 \mathrm{G}\right.$ and $\left.B_{1}=57.2 \mathrm{G}\right),{ }^{51}$ the isotropic hyperfine coupling constants of 56.8 and $17.5 \mathrm{MHz}$ (average of three tensors) lead to $\theta_{1}=-3.9 \pm 5$ with $\theta_{2}=-123.9 \pm 5$ and spin density of 0.35 at $C_{1}$. The resulting spin density at $C_{1}$ lies within the range of tyrosyl radicals found in photosystem II, ${ }^{46}$ and the tyrosyl model. ${ }^{45}$

Interaction of the Radical with the Hemes. To test whether the observed tyrosyl radical might be located in proximity of a heme center, we first checked for a possible broadening of the CW-EPR spectrum at 9-GHz. This is the most sensitive frequency to detect dipolar coupling in the EPR spectra as high-field spectra are rather dominated by $g$-strain. The simulations of the $70 \mathrm{~K}$ spectrum (Table 1) clearly indicated that the intrinsic line width at $9-\mathrm{GHz}$ was $5.6 \mathrm{G}$, which is typical for isolated tyrosyl radicals with unresolved 2,6-ring protons. We measured the

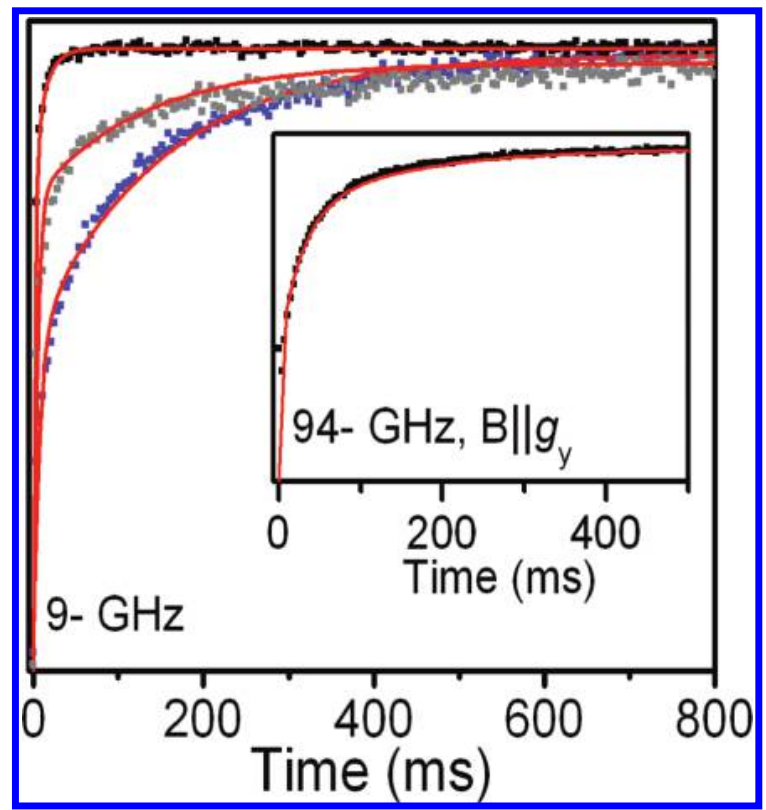

Figure 6. Inversion recovery of $9 \mathrm{GHz}$ recorded in the center of the tyrosyl radical spectrum at $6 \mathrm{~K}$ for PpoA reacted with 160 -fold $(8 R)$ HPODE for $7 \mathrm{~s}$ without (dashed black) and in presence of $20 \%$ glycerol (gray) and for the stable tyrosyl radical of subunit $\beta-2$ in class I RNR from E. coli (dashed blue). The curves were fitted to biexponential decays. Inset: $94-\mathrm{GHz}$ inversion recovery curves recorded at field position $B \| g_{y}$ at $6 \mathrm{~K}$ for a $7 \mathrm{~s}$ incubation PpoA sample with 160 -fold (8R)-HPODE (dashed black). The 94-GHz curve was best fitted with a sum of three exponentials.

CW-EPR spectrum also at $8 \mathrm{~K}$ using very low power $(200 \mathrm{nW})$. We found an identical line width to that observed at higher temperatures $(70 \mathrm{~K})$ and no evidence for additional broadening (data not shown).

Nevertheless, a weak dipolar interaction between the heme and the radical might be detected alternatively as an enhancement of the $T_{1}$ relaxation ${ }^{52}$ of the radical. Therefore, three-pulse inversion recovery experiments were performed and the curves best fitted to exponential decays. The short component arises predominately from spectral diffusion while the long component represents the electron spin-lattice relaxation time $\left(T_{1}\right)$ of the radical, which in case of anisotropic relaxation is orientation dependent. The relaxation rates at $6 \mathrm{~K}$ and $9-\mathrm{GHz}$ of the samples prepared on the millisecond to second time scale were of similar magnitude and ranged from $96-140 \mathrm{~s}^{-1}$. (8R)-HPODE and LA showed the same trends. The rates can be compared to that of an isolated tyrosyl radical. Hirsh et al. ${ }^{53}$ have previously measured relaxation rates at $9-\mathrm{GHz}$ for the stable tyrosyl radical of subunit $\beta$-2 in class I RNR from E. coli, which at low temperature has a relaxation rate near its intrinsic value (uncoupled). ${ }^{54}$ To ensure similar conditions we repeated the measurement on a sample of stable tyrosyl radical of subunit $\beta$ - 2 in class I RNR from E. coli and compared it to that of the tyrosyl radical in PpoA (both samples including the literature data contained $20 \%$ glycerol). The data are displayed in Figure 6. The rates obtained for the RNR sample $\left(1 / T_{1}=6.3 \mathrm{~s}^{-1}\right.$ at $\left.6 \mathrm{~K}\right)$ were well consistent with that previously recorded by using saturation recovery under similar conditions $\left(1 / T_{1}=7 \mathrm{~s}^{-1}\right.$ at $\left.6 \mathrm{~K}\right) .^{53}$ The results show a relaxation enhancement by a factor of 4 in PpoA as compared to the isolated radical. Finally, the relaxation rate was measured also at $94-\mathrm{GHz}$ and a value of $23 \mathrm{~s}^{-1}$ at $B \| g_{y}$ was found at $6 \mathrm{~K}$ (Figure 6, inset), which is 


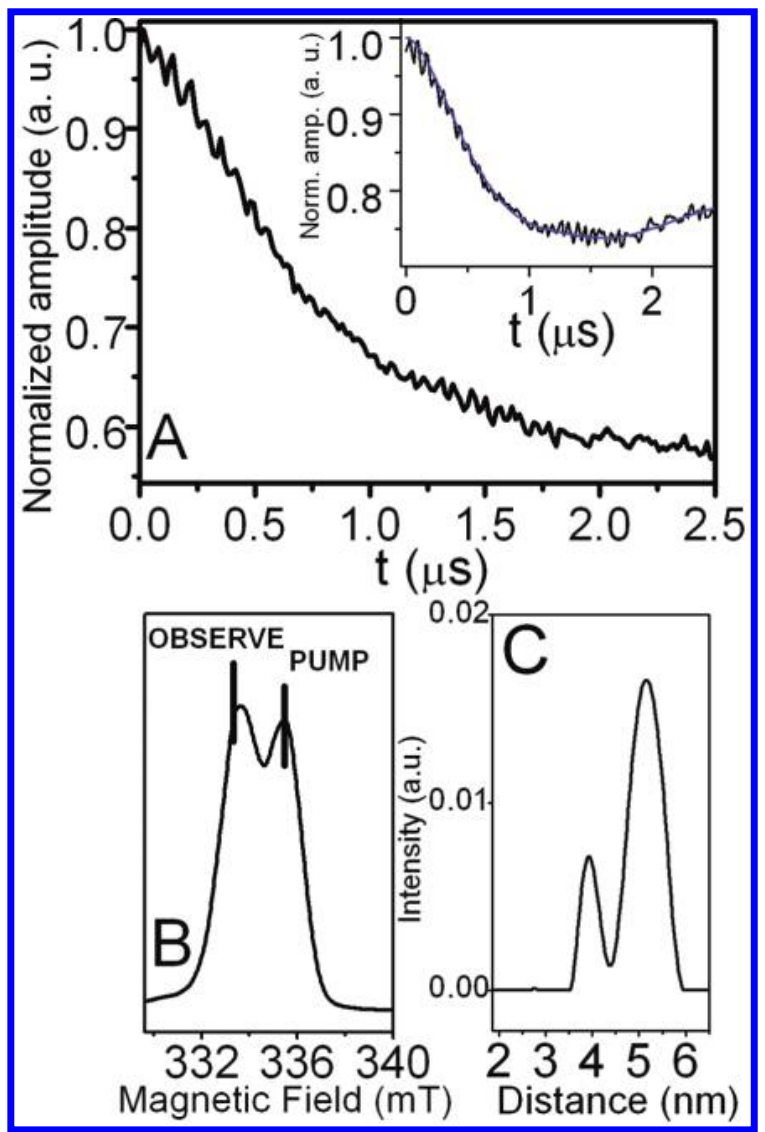

Figure 7. Representative data of a sample of PpoA aged for $7 \mathrm{~s}$ with 40 fold (8R)-HPODE. (A) Normalized four-pulse DEER trace at $5 \mathrm{~K}$. Pulses on the detection frequency $(\pi / 2, \pi)$ were 16 and $32 \mathrm{~ns} ; t_{\text {pump }}=$ $12 \mathrm{~ns} ; \tau_{1}=124 \mathrm{~ns} ; 44 \mathrm{~h}$ acquisition time. The spin-echo signal was observed on the low-field hyperfine line and the pump pulse was applied $65 \mathrm{MHz}$ away on the high-field line. Inset: Trace after subtraction of a monoexponential decay. Solid blue line is fit using distance-domain Tikhonov regularization. (B) Spin-echo detected spectrum of the radical signal. (C) Resulting distance distributions.

similar to that reported by Dorlet et al. ${ }^{55}$ at $140-\mathrm{GHz}$ for the radical in PGHS (29 $\left.\mathrm{s}^{-1}\right)$ and to the isolated tyrosyl radical in E. coli RNR $\left(\sim 40 \mathrm{~s}^{-1} \text { at } 140 \mathrm{GHz} \text { and } 8 \mathrm{~K}\right)^{56}$ Also the anisotropy of the relaxation rate at $94 \mathrm{GHz}$ and $6 \mathrm{~K}$ between $B \| g_{x}$ and $B \| g_{z}$ was found to be $25 \%$ and is similar to that of the uncoupled RNR tyrosyl radical. $^{56}$

Distance Measurements between Tyrosyl Radicals. The observation of some heterogeneity in the $g$-values and of a radical content higher than one radical per protein tetramer motivated us to examine the coexistence of a second or more tyrosyl radical species. For this purpose, we undertook DEER experiments, a technique that measures weak dipole-dipole interactions between the electron spins that span distances between approximately 15 and $80 \AA$. Previously, we have applied this technique to measure the distance between the two tyrosyl radicals on the monomeric R2 subunits in E. coli RNR. ${ }^{57}$ Using a similar experimental protocol, we performed the experiment at $9-\mathrm{GHz}$ on PpoA samples by mixing with 160 -fold (8R)-HPODE for $7 \mathrm{~s}$.

Figure 7A displays a typical echo modulation trace for a sample after $7 \mathrm{~s}$ of manual mixing. The time trace shows an oscillation, which is indicative of a long distance. To determine the tyrosyltyrosyl distance, the data were analyzed with the software
DeerAnalysis $2008 .^{33}$ After subtraction of a weak monoexponential decay, fitting using the distance-domain Tikhonov regularization procedure gave the distance distribution shown in Figure $7 \mathrm{C}$. A dominant peak is observed with a maximum at $5.2 \mathrm{~nm} \pm 0.4$ and a second of lower intensity at $3.9 \mathrm{~nm}$. The magnitude of the peak at $3.9 \mathrm{~nm}$ varied with different background subtractions. However, this high frequency modulation is apparent by visual inspection and is therefore not an artifact of the subtraction. A control experiment was performed, observing on the radical signal but pumping $65 \mathrm{MHz}$ away on the ferric heme signal so that only the heme could be excited by the pump pulse. The resulting trace showed no discernible modulation, indicating that we observe a radical-to-radical distance.

\section{DISCUSSION}

In this study we have characterized the paramagnetic centers in resting and active PpoA using CW and pulse EPR methods and rapid freeze-quenching techniques. Particularly, the results supported the proposal that the enzyme contains two different heme domains. Furthermore, we observed a new radical generated during reaction of PpoA with (8R)-HPODE and LA, respectively, in the millisecond time scale and concomitant to the depletion of the HS heme signal. The nature of the radical and its interaction with the other paramagnetic centers were investigated by pulsed EPR methods.

PpoA 9-GHz EPR spectra in the resting state exhibited two main resonances typical for both HS and LS ferric heme. The $g$-values of the LS heme are fingerprints for hemes with thiolate as the fifth ligand based on the substantive catalogue of experimentally determined values (Table S1). ${ }^{38,39}$ These LS $g$-values taken together with previous UV-vis data ${ }^{4}$ support the presence of a P450 heme thiolate domain as part of PpoA. On the other hand, the $g$-values of the HS heme (" $g=6$ ") signal are less informative about the type of heme binding domain associated with this signal. Indeed, $g$-values with $g \approx 6$ and $g \approx 2$ cannot be unequivocally assigned to a HS pentacoordinated ferric heme with a histidine ligand, which would be expected in the second, peroxidase-type binding domain proposed in PpoA. Furthermore, in Table $S 1$ we have summarized representative reported magnetic parameters of HS hemes. From this Table we note that also some P450 enzymes or model systems have revealed $g$-values similar to the ones found here in HS PpoA (see refs in Table S1). Therefore, the assignment of the HS spin signal to a second different heme domain is not feasible just based on the $g$-values. In order to examine the proposal in ref 4 that PpoA contains a peroxidase-type heme domain, ENDOR experiments were performed to assess the existence of an axial nitrogen coordination from a histidine to the HS heme. The ENDOR spectra recorded at $B \| g_{z}$ of the HS signal finally revealed typical resonances of an axial histidine ligand, based on comparison with other HS model systems, spectral simulations, and control experiments in $\mathrm{D}_{2} \mathrm{O}$. The result supports the previously reported sequence alignment ${ }^{4}$ suggesting $\mathrm{H} 377$ as the fifth ligand to a heme in a dioxygenase/peroxidase domain. Interestingly, we found also a second, weak LS signal with $g$-values characteristic for LS resonances as found in peroxidases ${ }^{58}$ and PGHS. ${ }^{13} \mathrm{We}$ attribute this signal to a small fraction of the peroxidase heme center that has a different configuration of the distal site.

During the time course of the reaction with ( $8 R)$-HPODE we have observed a substantial decrease of the HS heme on the millisecond time scale concomitant to the formation of a new radical. The results are consistent with the proposed first half 
reaction mechanism of Scheme 1 , in which (8R)-HPODE initiates the reaction in the peroxidase domain and suggests that the radical is produced in the first half reaction in the peroxidase domain. We were not able to discern any broad signal in the EPR spectra that could be attributed to a $\left[\mathrm{Fe}(\mathrm{IV})=\mathrm{O} \mathrm{Por}^{\bullet+}\right]$ (Compound I, Scheme 1). The porphyrin cationic radical $\left[\mathrm{Fe}(\mathrm{IV})=\mathrm{O} \mathrm{Por}^{\bullet+}\right]$ has been reported in the $g \approx 2$ and $g \approx 4$ spectral regions as a $S=3 / 2$ spin signal originating from the ferromagnetic coupling of the $\pi$-porphyrin radical and the ferryl ion. ${ }^{58-60}$ The signal was detectable only at low temperatures $(<10 \mathrm{~K})$ because of the strong interaction and short relaxation rates of the unpaired electron in close proximity to the heme iron. Noteworthy is that Compound I of a P450 enzyme has recently been detected under freeze-quench EPR conditions using $m$-chloroperbenzoic acid and freezing into liquid ethane at $89 \mathrm{~K}^{61}$ We suggest that $\left[\mathrm{Fe}(\mathrm{IV})=\mathrm{O} \mathrm{Por}^{\bullet+}\right]$ could be the precursor of the radical species, which we have not been able to observe thus far at the shortest detectable time point of $30 \mathrm{~ms}$ owing to a fast rate constant. Finally, the reduction in the intensity of the LS heme signal at later times (Figure S3) also suggests that a higher-valence intermediate species could be formed in this heme domain according to the proposed reaction mechanism (Scheme 1). Freeze-quench and UV-vis stopped flow studies are in progress to study this issue.

The detection of a radical formed during reaction of PpoA with the substrates supports the proposed mechanism that an amino acid radical is generated by reaction with ( $8 R)$-HPODE (Scheme 1). The line shape of the multifrequency EPR spectra provided a fingerprint for a tyrosyl radical. The high-field spectra display a large isotropic splitting at all three $g$-values superimposed with an anisotropic hyperfine substructure. The $g$-anisotropy $\left(g_{x}-g_{z}=0.0046\right)$ and the $g$-values are typical for tyrosyl radicals. ${ }^{45}$ The $g_{x}$-value of tyrosyl radicals is sensitive to the local electrostatic environment ${ }^{62-64}$ and to hydrogen bonding interactions, as was demonstrated in studies of $E$. coli RNR with $g_{x}=2.00912^{45}$ as compared to the hydrogen bonded radical in mouse RNR $\left(g_{x}=2.0076\right)^{47}$ and in other organisms as well as for the $\mathrm{Y}_{\mathrm{D}}$ radical of PS II with $g_{x}=2.0074 .^{46}$ The $g_{x}$-value found for the tyrosyl radical in PpoA $\left(g_{x}=2.0070\right)$ is consistent with an electropositive environment likely due to one or more hydrogen bonds or the presence of a nearby positively charged residue. Previous studies of tyrosyl radicals and model systems have shown that hydrogen bonds can strongly modify the stability and redox properties of the tyrosyl radical. ${ }^{65,66}$ The presence of a distribution in $g_{x}$ values has been documented previously in the HFEPR of protein based tyrosyl radicals. ${ }^{55}$ The heterogeneous environment at the phenolic oxygen can be thought to arise from the rearrangement of the tyrosine on oxidation in a hydrogen-bonding environment.

Experiments with the mutant Y374 were carried out to find more information about the possible radical site. Sequence alignments had suggested that residue Y374 is situated in a $\mathrm{N}$-terminal heme peroxidase domain. ${ }^{4}$ Circular dichroism had shown no evidence for changes in the folding of the enzyme caused by the mutation. ${ }^{4}$ Absence of a radical signal in the Y374F mutant indicates involvement of Y374 in the reaction mechanism. Y374 could be the radical site or part of a radical propagation pathway involving more than one tyrosine, as PpoA possesses a total of thirty-six tyrosines in each subunit of the tetramer. Such a well-known tyrosine pathway was established in $\mathrm{RNR}^{67}$ and recently more than one tyrosyl radical has been identified also in prostacyclin synthase on reaction with peracetic acid. ${ }^{68}$
Relaxation measurements were invoked to monitor the interaction between paramagnetic centers. A comparative experiment with E. coli RNR illustrated that the spin-lattice relaxation rate of the tyrosyl radical in PpoA is enhanced by a factor of 4 with respect to that of an uncoupled tyrosyl radical under identical conditions at 9-GHz, but no substantial difference (relaxation enhancement) was observed at 94-GHz as compared to the RNR E. coli data at $140-\mathrm{GHz}^{56}$ The result suggests that the interaction with a fast relaxing center, such a heme, is weak. The relaxation rates acquired during the course of the reaction ( $\mathrm{ms}$ to $\mathrm{s}$ ) were all similar within a range of $96-140 \mathrm{~s}^{-1}$. Finally, we have observed relaxation rates at $94-\mathrm{GHz}\left(1 / T_{1}=23\right.$ $\left.\mathrm{s}^{-1}, B \| g_{y}\right)$ similar to those reported for the tyrosyl radical in PGHS at $140-\mathrm{GHz}\left(1 / T_{1}=29 \mathrm{~s}^{-1}, B \| g_{y}\right)$ under similar conditions. $^{55}$ The similarity of the relaxation rates taken together with the sequence and mechanistic similarities between PGHS and the half reaction of PpoA suggests that the location of the tyrosyl radicals in PpoA and PGHS might be at a conserved tyrosine residue in the peroxidase domain.

The quantitation of the radical signal suggested that more than one radical is formed per tetramer. To provide evidence for this hypothesis we performed pulse EPR distance measurements. The DEER traces recorded by pumping and detecting on the tyrosyl radical signal showed a clear oscillation and gave direct evidence for the coupling between at least two tyrosyl species. The presence of another tyrosyl radical with similar spectral properties makes plausible the generation of the corresponding radical on another monomeric subunit of the tetrameric enzyme. Certainly, the dominant distance of $5.2 \mathrm{~nm}$ could place the interspin distance between two monomer units. We have also detected a second distance at $3.9 \mathrm{~nm}$ with a population of about $15-20 \%$ (depending on background subtraction). However, the weakness of this signal, in particular if related to the overall radical yield of 0.4 radical/tetramer, questions whether the signal is biochemically or mechanistically relevant.

Nevertheless, the detection of the dominant interspin distance unambiguously demonstrates the coexistence of more than one tyrosyl radical and is consistent with the hypothesis of a heterogeneous environment of the radicals reflected in the high-field EPR spectra ( $g_{x}$ broadening). We note that the formation of several tyrosyl radicals has been previously reported for prostacyclin synthase ${ }^{68}$ and Y504 has been attributed as an alternative radical site, ${ }^{69}$ which is one of four tyrosine residues conserved in PpoA making it a candidate for further mutagenesis studies. More detailed spectroscopic studies in combination with site specific isotopic labeling and kinetic studies will be required to assign the distances.

In conclusion, we have reported the EPR characterization of a novel fusion protein that uses two different domains to catalyze fatty acid hydroxylation. Our results acquired in the resting state and in the millisecond to second time scale of the enzymatic reaction gave evidence for a complex mechanism involving at least one heme domain and the formation of a tyrosyl radical species. The occurrence of several paramagnetic centers combined with the tetrameric structure of the enzyme led to the detection of several spin interactions, which indicated the radical to be in the vicinity of the heme domains and distributed on different monomer subunits of the enzyme. The results provide the basis for the design of future experiments to disentangle these interactions and locate the radical site using biochemical as well as spectroscopic methods such as site-specific replacement of amino acids, stopped flow UV-vis, and Mössbauer experiments. We also have illustrated the utility of DEER to provide a new 
parameter-the inter-radical distance-for locating amino acid radical species.

\section{ASSOCIATED CONTENT}

Supporting Information. Table S1 and Figures S1-S6. This material is available free of charge via the Internet at http:// pubs.acs.org.

\section{AUTHOR INFORMATION}

\section{Corresponding Author}

bennati@mpibpc.mpg.de

\section{ACKNOWLEDGMENT}

We thank Thomas F. Prisner for access to the $180-\mathrm{GHz}$ spectrometer; all 180-GHz spectra were recorded at J. W. Goethe Universität, Frankfurt, Germany. We thank Sun Un for initial experiments at 285-GHz. We are indebted to Yu Wang and Brigitta Angerstein for calibration of the freeze-quench apparatus and to Joanne Stubbe for a previous donation of E. coli R2 protein. We also thank Sabine Freitag for production of (8R)-HPODE and Thomas Nick for stimulating discussions. We acknowledge financial support of the DFG-IRTG 1422 and the Max Planck Society.

\section{ADDITIONAL NOTE}

${ }^{a}$ The $g$-values of LS rhombic ferric spectra have been used to correlate rhombicity and tetragonal ligand field strength terms in so-called "truth diagrams" by Blumberg and Peisach. ${ }^{39}$ These serve as a guide to assign the coordination environment of the heme. The derived crystal field parameters $(V / \lambda=4.67$ and $\Delta / \lambda=5.04)$ fall in group $\mathrm{P}$ of these diagrams, consistent with thiolate ligation.

\section{REFERENCES}

(1) Brodhun, F.; Feussner, I. FEBS I. 2011, 278, 1047-1063.

(2) Andreou, A.; Brodhun, F.; Feussner, I. Prog. Lipid Res. 2009, $48,148-170$.

(3) Funk, C. D. Science 2001, 294, 1871-1875.

(4) Brodhun, F.; Gobel, C.; Hornung, E.; Feussner, I. J. Biol. Chem. 2009, 284, 11792-11805.

(5) Tsitsigiannis, D. I.; Kowieski, T. M.; Zarnowski, R.; Keller, N. P. Eukaryot. Cell 2004, 3, 1398-1411.

(6) Tsitsigiannis, D. I.; Zarnowski, R.; Keller, N. P. J. Biol. Chem. 2004, 279, 11344-11353.

(7) Tsitsigiannis, D. I.; Kowieski, T. M.; Zarnowski, R.; Keller, N. P. Microbiologv 2005, 151, 1809-1821.

(8) Gilsenan, J. E. M.; Atherton, G.; Bartholomew, J.; Giles, P. F.; Attwood, T. K.; Denning, D. W.; Bowyer, P. Nucleic Acids Res. 2009, 37, D509-D514.

(9) Dotis, J.; Roilides, E. Int. I. Infect. Dis. 2004, 8, 103-110.

(10) Pihet, M.; Carrere, J.; Cimon, B.; Chabasse, D.; Delhaes, L.; Symoens, F.; Bouchara, J.-P. Med. Mvcol. 2009, 47, 387-397.

(11) Bhatnagar, D.; Yu, J.; Ehrlich, K. X. C. Chem. Immunol. 2002, 81, 167-206.

(12) Smith, W. L.; Marnett, L. J. Biochim. Biophys. Acta 1991, 1083, 1-17.

(13) Rouzer, C. A.; Marnett, L. I. Chem. Rev. 2003, 103, 2239-2304.

(14) (a) Tsai, A.-L.; Hsi, L. C.; Kulmacz, R. J.; Palmer, G.; Smith, W. L. I. Biol. Chem. 1994, 269, 5085-5091. (b) Tsai, A.-L.; Kulmacz, R. J. Arch. Biochem. Biophys. 2010, 493, 103-124.
(15) Goodwin, D. C.; Gunther, M. R.; Linda, C. H.; Crews, B. C.; Eling, T. E.; Mason, R. P.; Marnett, L. J. J. Biol. Chem. 1998, 273, 8903-8909.

(16) Shimokawa, T.; Kulmacz, R. J.; DeWitt, D. L.; Smith, W. L. L. Biol. Chem. 1990, 265, 20073-20076.

(17) Su, C.; Sahlin, M.; Oliw, E. H. J. Biol. Chem. 1998, 273, 20744-20751.

(18) Stubbe, J.; van der Donk, W. A. Chem. Rev. 1998, 98, 705-762.

(19) Sjöberg, B.-M.; Gräslund, A. Adv. Inorg. Biochem. 1983, $5,87-110$.

(20) Barry, B. A.; Babcock, G. T. Proc. Natl. Acad. Sci. U.S.A. 1987, 84, 7099-7103.

(21) (a) Singh, R.; Switala, J.; Loewen, P. C.; Ivancich, A. J. Am. Chem. Soc. 2007, 129, 15954-15963. (b) Fielding, A. J.; Singh, R.; Boscolo, B.; Loewen, P. C.; Ghibaudi, E.; Ivancich, A. Biochemistry 2008, 47, 9781-9792. (c) Zhao, X.; Suarez, J.; Khajo, A.; Yu, S.; Metlitsky, L.; Magliozzo, R. I. Am. Chem. Soc. 2010, 132, 8268-8269.

(22) Koeduka, T.; Matsui, K.; Akakabe, Y.; Kajiwara, T. J. Biol. Chem. 2002, 277, 22648-22655.

(23) (a) Gupta, A.; Mukherjee, A.; Matsui, K.; Roth, J. P. J. Am. Chem. Soc. 2008, 130, 11274-11275. (b) Mukherjee, A.; Angeles-Boza, A. M.; Huff, G. S.; Roth, J. P. I. Am. Chem. Soc. 2011, 133, 227-238.

(24) Bennati, M.; Prisner, T. F. Rep. Prog. Phys. 2005, 68, 411-448.

(25) Williams-Smith, D. L.; Bray, R. C.; Barber, M. J.; Tsopanakis, A. D.; Vincent, S. P. Biochem. I. 1977, 167, 593-600.

(26) Ballou, D. P.; Palmer, G. A. Anal. Chem. 1974, 46, 1248-1253.

(27) Aasa, R.; Vänngard, T. L. Magn. Reson. 1975, 19, 308-315.

(28) Stoll, S.; Schweiger, A. I. Magn. Reson. 2006, 178, 42.

(29) Milov, A. D.; Ponomarev, A. B.; Tsvetkov, Y. D. Chem. Phys. Lett. 1984, 110, 67.

(30) Milov, A. D.; Maryasov, A. G.; Tsvetkov, Y. D. Appl. Magn. Reson. 1998, 15, 107.

(31) Jeschke, G. Macromol. Rapid Commun. 2002, 23, 227.

(32) Pannier, M.; Veit, S.; Godt, A.; Jeschke, G.; Spiess, H. W. L.Magn. Reson. 2000, 142, 331-340.

(33) Jeschke, G.; Chechik, V.; Godt, A.; Zimmermann, H.; Banham, J.; Timmel, C. R.; Hilger, D.; Jung, H. Appl. Magn. Reson. 2006, 30, 473.

(34) Bennati, M.; Farrar, C. T.; Bryant, J. A.; Inati, S. J.; Weis, V.; Gerfen, G. J.; Riggs-Gelasco, P.; Stubbe, J.; Griffin, R. G. J. Magn. Reson. 1999, 138, 232-243.

(35) Hertel, M. M.; Denysenkov, V. P.; Bennati, M.; Prisner, T. F. Magn. Reson. Chem. 2005, 43, S248-S255.

(36) Burghaus, O.; Plato, M.; Rohrer, M.; Mobius, K.; Macmillan, F.; Lubitz, W. L.Phvs. Chem. 1993, 97, 7639-7647.

(37) Smith, D. T.; Pilbrow, J. R. In Biological Magnetic Resonance; Berliner, L. J., Reuben, J., Eds.; Plenum, New York: 1980, p 85.

(38) Walker, F. A. Coord. Chem. Rev. 1999, 185-186, 471-534.

(39) Blumberg, W. E.; Peisach, J. In Probes and Structure and Function of Macromolecules and Membranes; Chance, B.; Yonetani, T.; Mildvan, A. S., Eds; Academic Press, New York, 1971, Vol. 2; pp 215-229.

(40) Peisach, J.; Blumberg, W. E.; Adler, A. Ann. N.Y. Acad. Sci. 1973, 206, 310-327.

(41) Scholes, C. P.; Lapidot, A.; Mascarenhas, R.; Inubushi, T.; Isaacson, R. A.; Feher, G. L.Am. Chem. Soc. 1982, 104, 2724-2735.

(42) Jiang, F. S.; Zuberi, T. M.; Cornelius, J. B.; Clarkson, R. B.; Gennis, R. B.; Belford, R. L. L. Am. Chem. Soc. 1993, 115, 10293-10299.

(43) Garcia-Rubio, I.; Braun, M.; Gromov, I.; Thony-Meyer, L.; Schweiger, A. Biophvs. I. 2007, 92, 1361-1373.

(44) Fittipaldi, M.; Garcia-Rubio, I.; Trandafir, F.; Gromov, I.; Schweiger, A.; Bouwen, A.; Van Doorslaer, S. J. Phys. Chem. B 2008, 112, 3859-3870.

(45) Gerfen, G. J.; Bellew, B. F.; Un, S.; Bollinger, J. M.; Stubbe, J.; Griffin, R. G.; Singel, D. J. I. Am. Chem. Soc. 1993, 115, 6420.

(46) Un, S.; Dorlet, P.; Rutherford, A. W. Appl. Magn. Reson. 2001, $21,341$.

(47) Schmidt, P.; Andersson, K.; Barra, A.-L.; Thelander, L.; Gräslund, A. I. Biol. Chem. 1996, 271, 23615-23618.

(48) McLachlan, A. D. Mol. Phvs. 1958, 1, 233-240. 
(49) Lykos, P. G. L. Chem. Phys. 1960, 32, 625-626.

(50) Heller, H.; McConnell, H. M. I. Chem. Phvs. 1960, 32, $1535-1539$.

(51) Fessenden, R. W.; Schuler, R. H. I. Chem. Phys. 1963, 39, 2147-1333.

(52) Berliner, L. J.; Eaton, G. R.; Eaton, S. S. Distance Measurements in Biological Systems by EPR, Kluwer, New York, 2000.

(53) Hirsh, D. J.; Beck, W. F.; Lynch, J. B.; Que, L., Jr.; Brudvig, G. W. I. Am. Chem. Soc. 1992, 114, 7475-7481.

(54) Sahlin, M.; Petersson, L.; Gräslund, A.; Ehrenberg, A.; Sjöberg, B.-M.; Thelandert, L. Biochemistry 1987, 26, 5541-5548.

(55) Dorlet, P.; Seibold, S. A.; Babcock, G. T.; Gerfen, G. J.; Smith, W. L.; Tsai, A.-L.; Un, S. Biochemistry 2002, 41, 6107-6114.

(56) Bar, G.; Bennati, M.; Nguyen., H. H. T.; Ge, J.; Stubbe, J.; Griffin, R. G. I. Am. Chem. Soc. 2001, 123, 3569-3576.

(57) Bennati, M.; Weber, A.; Antonic, J.; Perlstein, D. L.; Robblee, J.; Stubbe, J. A. I. Am. Chem. Soc. 2003, 125, 14988-14989.

(58) Dunford, H. B. Heme Peroxidases; Wiley-VCH, John Wiley and Sons: New York, 1999.

(59) Schulz, C. E.; Devaney, P. W.; Winkler, H.; Debrunner, P. G.; Doan, N.; Ching, R.; Rutter, R.; Hager, L. P. FEBS Lett. 1979, 103, $102-105$.

(60) Singh, R.; Berry, R. E.; Yang, F.; Zhang, H. J.; Walker, F. A .; Ivancich, A. Biochemistry 2010, 49, 8857-8872.

(61) Rittle, J.; Green, M. T. Science 2010, 330, 933-937.

(62) Un, S.; Atta, M.; Fontecave, M.; Rutherford, A. W. J. Am. Chem. Soc. 1995, 117, 10713-10719.

(63) Un, S.; Gerez, C.; Elleing, E.; Fontecave, M. J. Am. Chem. Soc. 2001, 123, 3048-3054.

(64) Engstrom, M.; Himo, F.; Gräslund, A.; Minaev, B.; Vahtras, O.; Agren, H. L. Phvs. Chem. A 2000, 104, 5149-5153.

(65) Hoganson, C. W.; Tommos, C. Biochim. Biophys. Acta, Bioenerg. 2004, 1655, 116-122.

(66) Nara, S. J.; Valgimigli, L.; Pedulli, G. F.; Pratt, D. A J. Am. Chem. Soc. 2010, 132, 863-872.

(67) Stubbe, J.; Nocera, D. G.; Yee, C. S.; Chang, M. C. Chem. Rev. 2003, 103, 2167-2201.

(68) Yeh, H. C.; Gerfen, G. J.; Wang, J. S.; Tsai, A.-L.; Wang, L. H. Biochemistry 2009, 48, 917-928.

(69) Rogge, C. E.; Liu, W.; Wu, G.; Wang, L.; Kulmacz, R. J.; Tsai, A.-L. Biochemistry 2004, 43, 1560-1568. 